\title{
VARIABILITY OF LUMINOUS STARS IN THE LARGE MAGELLANIC CLOUD USING 10 YEARS OF ASAS DATA
}

\author{
D. M. Szczygię ${ }^{1}$, K. Z. Stanek ${ }^{1}$, A. Z. Bonanos ${ }^{2}$, G. Pojmański ${ }^{3}$, B. Pilecki ${ }^{3}$, and J. L. Prieto ${ }^{4,5}$ \\ ${ }^{1}$ Department of Astronomy, The Ohio State University, 140 West 18th Avenue, Columbus, OH 43210, USA; szczygiel@astronomy.ohio-state.edu, \\ kstanek@astronomy.ohio-state.edu \\ ${ }^{2}$ Institute of Astronomy \& Astrophysics, National Observatory of Athens, I. Metaxa \& Vas. Pavlou St., P. Penteli, 15236 Athens, Greece; bonanos@ astro.noa.gr \\ ${ }^{3}$ Warsaw University Astronomical Observatory, Al. Ujazdowskie 4, 00-478 Warsaw, Poland;, gp@ astrouw.edu.pl, pilecki@astrouw.edu.pl \\ ${ }^{4}$ Carnegie Observatories, 813 Santa Barbara Street, Pasadena, CA 91101, USA; jose@ obs.carnegiescience.edu \\ Received 2010 February 1; accepted 2010 April 20; published 2010 May 18
}

\begin{abstract}
Motivated by the detection of a recent outburst of the massive luminous blue variable LMC-R71, which reached an absolute magnitude $M_{V}=-9.3 \mathrm{mag}$, we undertook a systematic study of the optical variability of 1268 massive stars in the Large Magellanic Cloud, using a recent catalog by Bonanos et al. as the input. The ASAS All Star Catalog provided well-sampled light curves of these bright stars spanning 10 years. Combining the two catalogs resulted in 599 matches, on which we performed a variability search. We identified 117 variable stars, 38 of which were not known before, despite their brightness and large amplitude of variation. We found 13 periodic stars that we classify as eclipsing binary (EB) stars, 8 of which are newly discovered bright massive EBs composed of OBtype stars. The remaining 104 variables are either semi- or non-periodic, the majority (85) being red supergiants (RSGs). Most (26) of the newly discovered variables in this category are also RSGs with only three B and four O stars.
\end{abstract}

Key words: binaries: eclipsing - Magellanic Clouds - stars: massive - stars: variables: general

Online-only material: color figures, machine-readable and VO tables

\section{INTRODUCTION}

Studies of stellar variability in the Magellanic Clouds have a long and venerable history, starting with a 1904 Harvard College Observatory Circular by E. C. Pickering stating that "The two Magellanic Clouds have long been objects of careful study, on account of the extraordinary physical conditions which prevail in them. They have not, however, heretofore been known as regions in which variable stars are numerous" (Pickering 1904, p. 1). That circular, although formally authored by Pickering, in fact reported 152 new variable stars in the Large Magellanic Cloud found by Henrietta Leavitt, work that was soon followed by "843 New Variable Stars in the Small Magellanic Cloud" (Leavitt \& Pickering 1905), "1777 Variables in the Magellanic Clouds" (Leavitt 1908), and culminated in the formulation of the Cepheid period-luminosity relation (the "Leavitt Law"; Leavitt \& Pickering 1912), a result of fundamental and continued importance in astronomy and cosmology (e.g., Freedman et al. 2001; Macri et al. 2006).

Later studies of stellar variability in the Clouds included many papers on Cepheids (e.g., Caldwell \& Coulson 1986; Madore \& Freedman 1991), but there were also studies of long-period variables (e.g., Wood et al. 1983; Feast et al. 1989), irregular variables of the S Doradus type (luminous blue variables, LBVs; e.g., Stahl et al. 1983), and RR Lyr stars (e.g., Thackeray 1958; Walker 1992), just to name the most popular subjects. By virtue of being so close to us and being relatively unobscured, the Magellanic Clouds continue to provide an ideal terrain for stellar variability studies.

The idea of Paczyński (1986) to use the Magellanic Clouds as targets for microlensing surveys to detect dark matter in the form of MACHOs has led to a new and glorious era in variability

\footnotetext{
5 Hubble and Carnegie-Princeton Fellow.
}

studies in general, not just in the Clouds, and has resulted in a wide variety and large number of papers reporting on variability in the Large Magellanic Cloud (LMC) and the Small Magellanic Cloud (SMC), including work on Cepheids (e.g., Alcock et al. 1995; Udalski et al. 1999; Soszyński et al. 2008), eclipsing binaries (EBs; e.g., Alcock et al. 1997; Wyrzykowski et al. 2004), RR Lyr stars (e.g., Alcock et al. 1996; Soszyński et al. 2003, 2009), all kinds of stellar variability (e.g., Cook et al. 1995; Żebruń et al. 2001), and even quasars (e.g., Geha et al. 2003; Dobrzycki et al. 2003; Kozłowski et al. 2010), supernovae (e.g., Garg et al. 2007) behind the Clouds and supernovae light echoes in the LMC (Rest et al. 2005). Some microlensing events were also found in the Clouds, but clearly not enough to populate the halo of our Galaxy with MACHOs (e.g., Alcock et al. 2000; Tisserand et al. 2007; Wyrzykowski et al. 2009).

Given all that tremendous effort and many hundreds of papers written about stellar variability in the Magellanic Clouds, is there still room for more discoveries? As is almost always the case in astronomy, the answer is yes. One path is simply to go deeper, and some impressive results have been reported recently, such as the detection of extragalactic W UMa binaries in the LMC (Kałużny et al. 2006) using ground-based data from a largeaperture telescope. Another path is to find variable stars in the densest parts of the Clouds, which are now accessible using the Hubble Space Telescope (e.g., Fiorentino et al. 2008). Still yet another path is to take advantage of the fact that we have been observing stars in the Magellanic Clouds for a long time now (e.g., Kozłowski et al. 2010), even as long as a hundred years (Grindlay et al. 2009). But the path taken in this paper, namely to go shallower, and to cover all of the Clouds (the SMC will be discussed in a future paper), again owes its existence to Bohdan Paczyński, whose vision also led to the All Sky Automated Survey (ASAS) project led by Grzegorz Pojmański, who created it and has kept it functional for the past 10 years. 


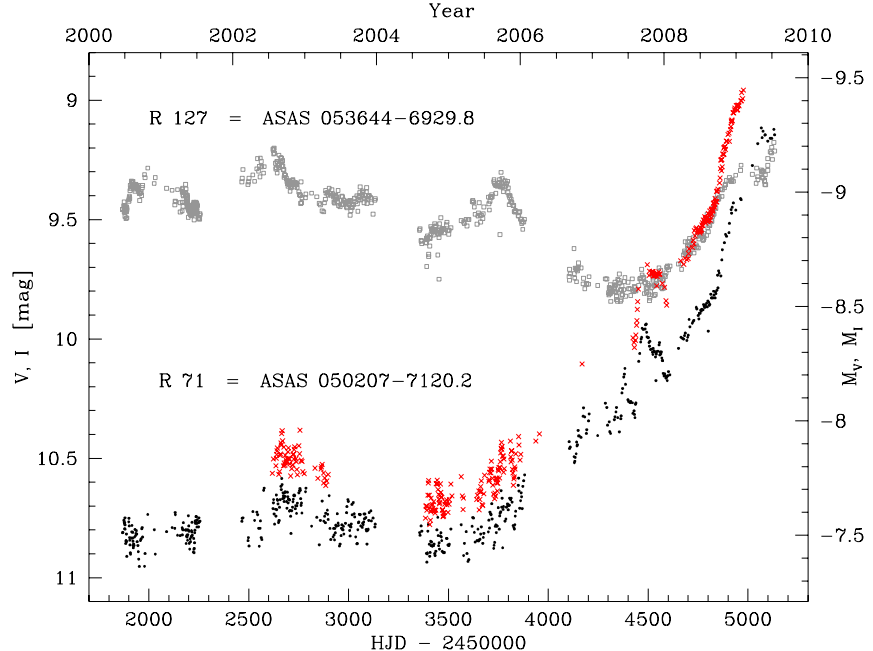

Figure 1. ASAS light curves of the LBVs R 71 in $V$ (black circles) and $I$ (red crosses) bands, and R 127 in $V$ (gray open squares), spanning 10 years. The right-hand axis shows the absolute magnitude value, assuming an LMC distance modulus of 18.41 mag (Macri et al. 2006).

(A color version of this figure is available in the online journal.)

A particular motivation for our study was provided by the recently reported outburst of an LMC LBV R71, ${ }^{6}$ which during the last five years has brightened by more than 2 mag to a visual magnitude of about 9.0, becoming the brightest star in the LMC (Gamen et al. 2009). As it turns out, the ASAS (Pojmański 1997, 2002; Pojmański et al. 2005) photometric database contained a detailed $V$-band light curve of that star (see Figure 1), obtained and reported in an automatic fashion by ASAS. Motivated by the fact that the brightening of R71 started several years ago, but was first reported only in 2008, we decided to investigate light curves of other bright stars in the LMC, using the ASAS data. R127 is another example of a ninth magnitude LBV in the LMC that underwent dramatic change starting in 2005 that was recorded by $\mathrm{ASAS}^{7}$ (see Figure 1), and was not noticed until two years later by Walborn et al. (2008), who began monitoring it spectroscopically. There are bound to be other such examples of bright LMC variables already recorded in the ASAS database. We therefore used the recent catalog of Bonanos et al. (2009) as an input catalog to characterize the variability properties of these known LMC massive stars. Confirming our suspicion, there are not only many spectacular light curves among known variables, but also a significant fraction of new interesting objects.

This paper has the following structure. In Section 2, we briefly describe both the input catalogs and the matching procedure. Section 3 introduces our variable star selection methods and discusses the identified variables. In Section 4, we present color-magnitude diagrams (CMDs) of the variable stars and discuss special cases. We summarize this work in Section 5.

\section{DATA}

\subsection{Input Catalogs}

We use the catalog of Bonanos et al. (2009, hereafter B09) as the source of bright, massive objects associated with the LMC. It contains photometric measurements for 1268 massive stars in several visual and infrared wavelengths $\left(U B V I, J H K_{S}\right.$, and 3.6-24 $\mu \mathrm{m})$, as well as their spectral classifications. The area

\footnotetext{
R71 = ASAS 050207-7120.2.

7 R127 = ASAS 053644-6929.8 (although blended).
}

covered by this catalog is delimited by $-72^{\circ}<\delta<-64^{\circ}$ and $70^{\circ}<\alpha<90^{\circ}$.

The $\operatorname{ASAS}^{8}$ (Pojmański 2002) has been monitoring the southern sky $\left(\delta<+28^{\circ}\right)$ since 2000 and by now the average number of data points per star in the LMC region is 550 in the $V$ band and 350 in the $I$ band. The $V$ magnitude range covered by the ASAS telescopes is around 8-14.5, which ideally suits a study of the brightest objects in the LMC. The number of objects visible in the LMC direction, within the coordinates listed above, is about 28,000, many of them being Galactic field stars.

\subsection{Matching Procedure}

The main difficulty in combining the two catalogs resulted from the large size of the ASAS pixel, which is $\sim 14^{\prime \prime} .9$. Thus, even though coordinates of objects in the ASAS catalogs are accurate to $\sim 3^{\prime \prime}$ (Pojmański 1997) and the positions within the B09 catalog are typically accurate to less than $\sim 1^{\prime \prime}$, in very crowded regions one ASAS object may be composed of more than one (bright) LMC star.

For this reason, we performed catalog matching in two steps. First, each of the 1268 stars from the B09 catalog was assigned one closest counterpart from the ASAS catalog (28,000 sources), without putting any constraints on counterpart separation. Among 1268 matching entries, 761 turned out to be unique, which means that an ASAS star in such a pair was assigned only one counterpart from the B09 catalog. Within the remaining 507 matching entries, one ASAS star was assigned to either two or three B09 stars and as a result these 507 matching entries contained only 162 unique ASAS sources. This was the case in the densest regions of the LMC, where due to low ASAS resolution objects from the B09 catalog could not be resolved.

In the second step, we took the $923(761+162)$ ASAS stars selected in the previous step and checked how many B09 neighbors each ASAS star had within a radius of $15^{\prime \prime}(1)$, between radii $15^{\prime \prime}$ and $30^{\prime \prime}$ (2), and between $30^{\prime \prime}$ and $45^{\prime \prime}$ (3). Given that the aperture radius adopted by us is in most cases $(\sim 70 \%) 1$ ASAS pixel, we assumed that the match could be considered unambiguous when there was only one neighbor within (1) and none within (2). There were 675 objects fulfilling this criterion. However, for about $30 \%$ of ASAS stars we have adopted apertures greater than 1 pixel, which could result in neighboring stars overlapping each other, so we decided to apply a more conservative criterion that no stars were allowed in either zone (2) or (3). The number of matches was reduced to 599 , which we accepted as our final sample. The choice of the aperture size described above is automatic and depends only on the magnitude of an object-for the faintest stars (13-14 mag) we use the smallest aperture radius of 1 pixel, while for the brightest $(8 \mathrm{mag}$ ) we use the largest aperture radius of 3 pixels.

It is important to stress here that the match has been made only against the B09 catalog, which means that the foreground field stars are not accounted for. At the same time, the B09 catalog itself is far from a complete list of massive stars in the LMC, as it contains only the stars for which spectra exist. Therefore, we should expect some crowding problems even in our final sample. This issue is addressed in Section 3.3.

The spatial distribution of the 599 matched objects in equatorial coordinates is presented in Figure 2 with black filled and open circles. Gray dots represent all ASAS objects in this region brighter than $V=14 \mathrm{mag}$ ( $\sim 19,000$ of $\sim 28,000$ points). The sample is dominated by foreground stars.

\footnotetext{
8 http://www.astrouw.edu.pl/asas
} 


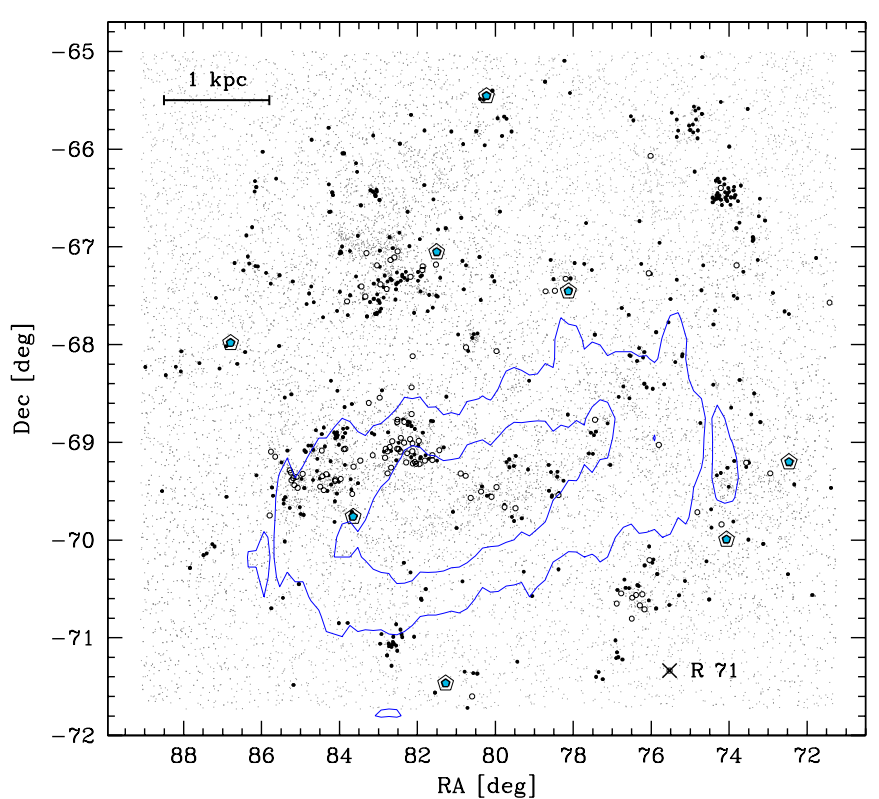

Figure 2. Spatial distribution of ASAS stars in the LMC region in equatorial coordinates. Gray dots are the $\sim 19,000$ objects brighter than $V=14$ mag (out of $\sim 28,000$ total) found by ASAS in this region of the sky. Filled circles represent the 599 final matches between the ASAS and B09 catalogs and open circles are the subset that was identified as variable (117 stars). Blue pentagons mark new EB candidates (see Section 3 for details). To show the location of the LMC bar, we also plot the stellar density contours based on the OGLE-III photometric maps of the LMC (Udalski et al. 2008). We only include $I<15$ mag objects, binned into $0.2 \mathrm{deg}$ regions in both R.A. and decl. The contour levels are 50 (for the outer) and 90 (for the inner) stars per bin. The outer contour includes the 30 Doradus star-forming region. Note that the OGLE fields do not cover the Shapley Constellation III star-forming region. The $1 \mathrm{kpc}$ marker is based on $18.41 \mathrm{mag}$ LMC distance modulus (Macri et al. 2006).

(A color version of this figure is available in the online journal.)

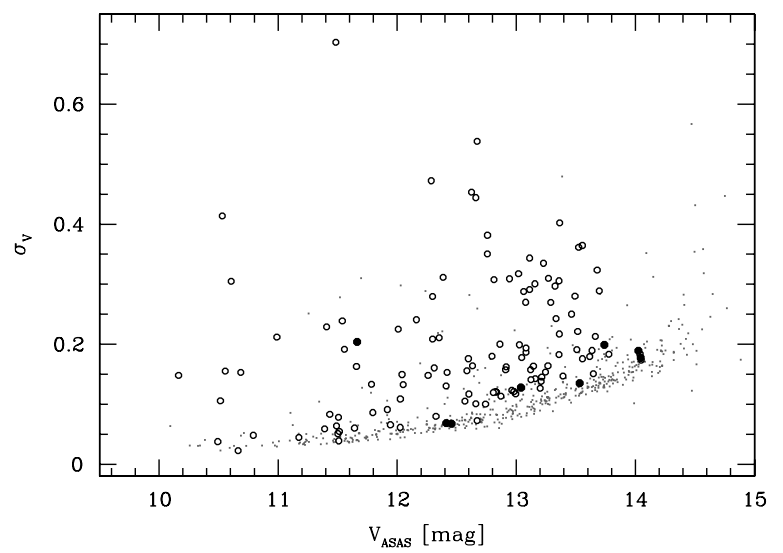

Figure 3. Standard deviation $\sigma_{\mathrm{V}}$ vs. $V$-band mean magnitude for all 599 matched sources. Variable stars are marked with open circles; EB candidates (eight points) are denoted by filled circles (see Section 3.1 for details).

\section{RESULTS OF VARIABLE STAR SEARCH}

As already summarized in the Introduction, the LMC has been the target of variability searches since the beginning of the last century, with an increasing intensity over the last two decades. Recent catalogs of variable stars were mainly generated using the data acquired by microlensing surveys such as OGLE and MACHO. However, these catalogs are complete for stars fainter than 13-14 $V$ magnitude, and are missing the brightest objects. This oversight can be corrected with the aid of ASAS data.
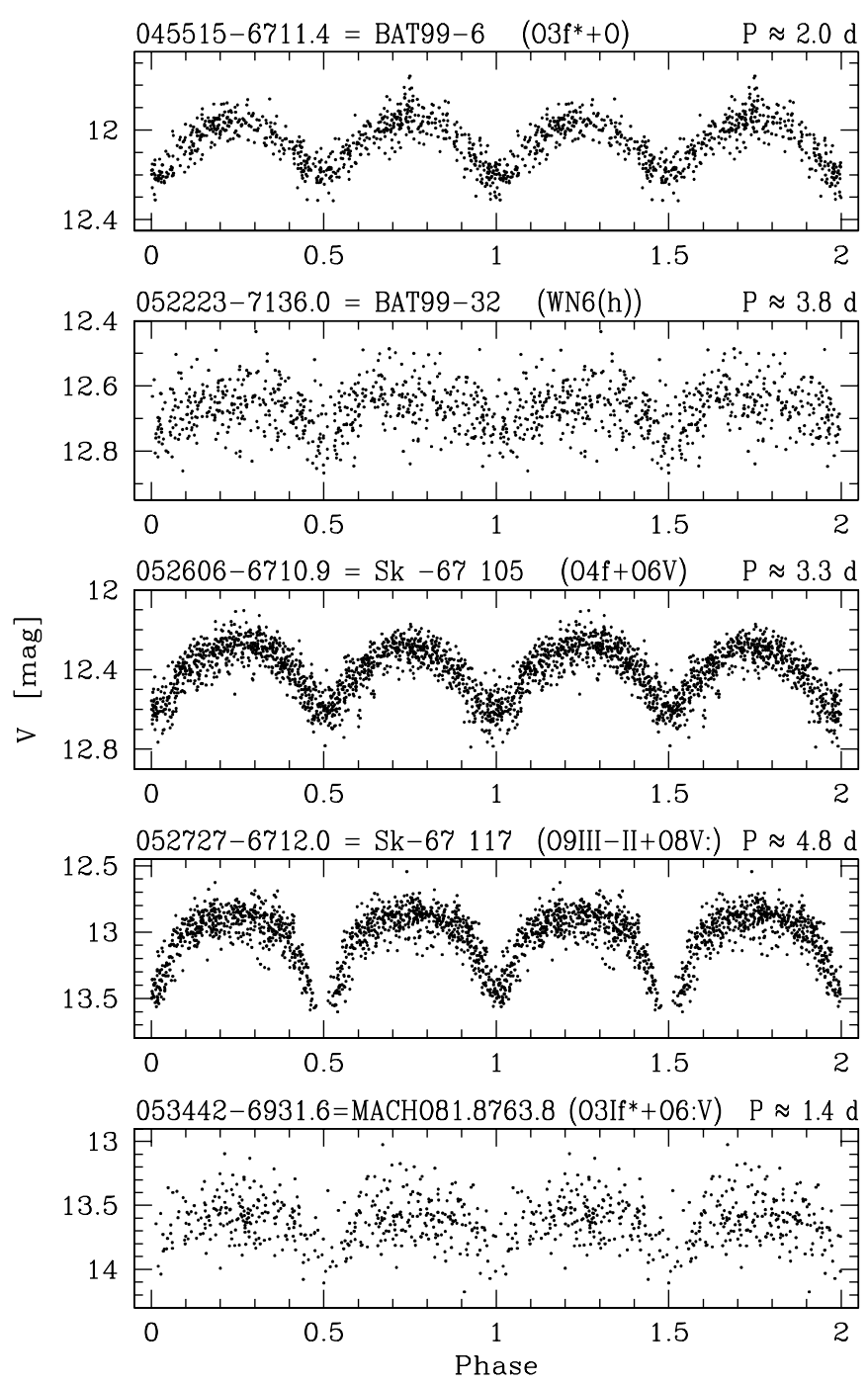

Figure 4. Phased ASAS $V$-band light curves of the five previously known EBs, sorted by right ascension, spanning 10 years. The header of each light curve contains two IDs, spectral type (in brackets), and orbital period in days. For more information on these stars, see Table 1.

\subsection{Variable Star Selection}

In order to investigate variability of the 599 matched stars, we extracted $V$-band light curves from the online ASAS database, keeping only points with quality flag "A." Then we cleaned the light curves by removing points that lie more than $3 \sigma$ from a local linear model fitted to each light curve, where $\sigma$ is the standard deviation. All cleaned light curves were subject to variability analysis using the analysis of variances (AoV) algorithm (Schwarzenberg-Czerny 1989) in the frequency range 0.0001-8 cycles per day. Each light curve was then folded with a period equivalent to the frequency with the highest peak in the power spectrum and visually inspected to eliminate possible spurious detections such as 1 day aliases. During the visual inspection, other frequencies with high signal were examined and the best period was chosen.

Figure 3 presents the standard deviation $\sigma_{\mathrm{V}}$ versus $V$-band mean magnitude with all 599 matched sources marked with gray dots and variable stars with larger circles. There are a number of stars with high $\sigma_{\mathrm{V}}$ values that were not classified as variable. These objects have an increase in brightness around HJD 2452200 and 2452600 which was due to strong defocusing of the ASAS telescopes. 

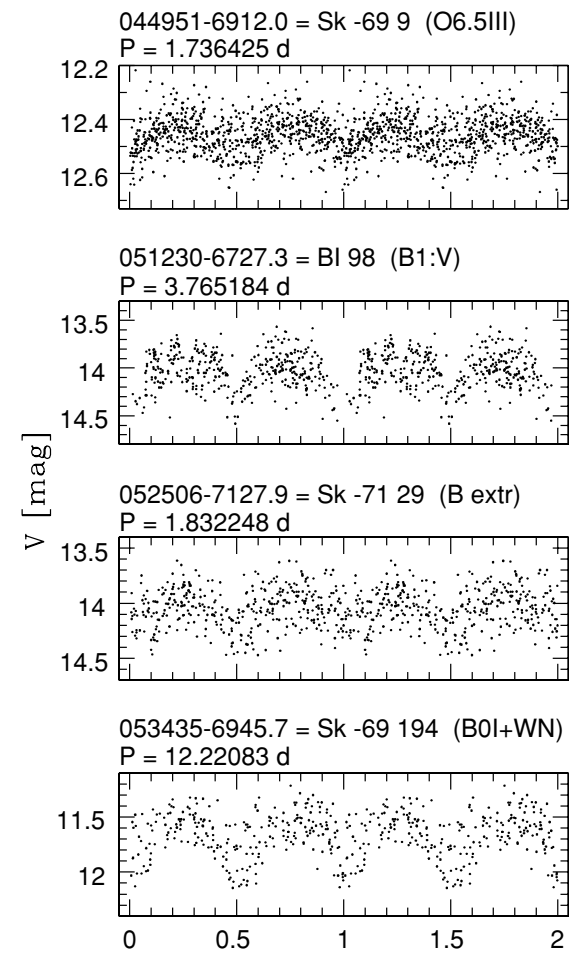

Phase

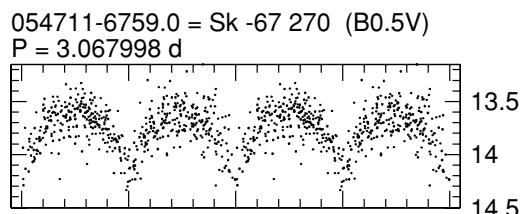

052601-6703.1 = Sk -67 102 (B2III SB:) $P=3.659535 d$

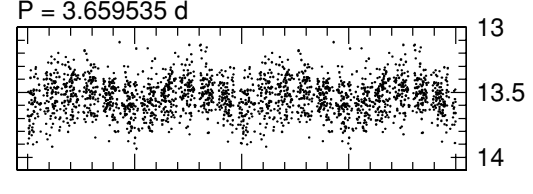

052055-6527.3 = Sk -65 47 (O4If) $P=3.701956 \mathrm{~d}$

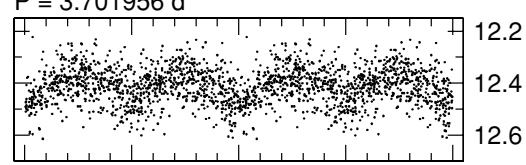

045617-6959.6 = Sk -70 18 (B0n) $P=7.625148 d$



Figure 5. Phased ASAS V-band light curves of eight new EB candidates, sorted by right ascension. The header of each light curve contains two IDs, spectral type (in brackets), and orbital period in days. For more information on these stars, see Table 2.

During the inspection process we identified 117 variable stars, of which 55 were not listed as variable in the $\operatorname{Simbad}^{9}$ database. We then matched these 55 stars against the General Catalogue of Variable Stars (GCVS; Samus et al. 2009) using VizieR ${ }^{10}$ and found that another 24 were known to be variable, which altogether gives 79 known variable stars and 38 new discoveries.

\subsection{Periodic Variables}

Among our 117 detections there are 13 periodic variables; 5 of them are known binary systems (although BAT99-32 was only known as a spectroscopic binary and its eclipsing nature has not been reported before), which are as follows.

1. 045515-6711.4 = BAT99-6 = Brey $5=$ Sk -6718 (Prevot-Burnichon et al. 1981): Seggewiss et al. (1991) have identified this object as a binary system with a tentative period of 1.99 days, based on a very poor light curve. Later Niemela et al. (2001) have shown it to be "a multiple system consisting of at least two pairs of short-period binaries." We confirm the 2 day period of the eclipsing pair.

2. 052223-7136.0 = BAT99-32: a long known Wolf-Rayet (W-R) star (Westerlund \& Smith 1964), which has been reported to be a single-lined spectroscopic binary (Moffat 1989 ) and an EB with a period of $\sim 1.91$ days (Seggewiss et al. 1991). The discovery from ASAS observations, that it is also an eclipsing system with a period $P \sim 3.8$ days that is twice as long as that found in the literature, explains the low radial velocity amplitude and unusually short period previously reported. High-resolution spectra of this system would be very valuable as they would allow for a determination of its parameters, given that very few W-R stars have good measurements of masses and radii.

\footnotetext{
9 http://simbad.u-strasbg.fr

$10 \mathrm{http}: / /$ vizier.u-strasbg.fr
}

3. 052606-6710.9 = Sk -67 105: Niemela \& Morrell (1986) found it to be a massive double-lined spectroscopic binary (the first extragalactic binary to be studied), while Haefner et al. (1994) discovered it is a near contact eclipsing system. Ostrov \& Lapasset (2003) derived component masses of $48.3 M_{\odot}$ and $31.4 M_{\odot}$.

4. $052727-6712.0=\mathrm{Sk}-67117=\mathrm{HV}$ 2543: a known semidetached double-lined EB with masses $25.63 \pm 0.7 M_{\odot}$ and $15.63 \pm 1.0 M_{\odot}$ (Niemela \& Bassino 1994; Ostrov et al. 2000).

5. 053442-6931.6 = MACHO81.8763.8: discovered as an EB by Alcock et al. (1997), it was shown to be a massive binary with component masses of $41 M_{\odot}$ and $27 M_{\odot}$ by Ostrov (2001).

The $V$-band light curves of these binaries, spanning 10 years of ASAS observations, are plotted in Figure 4 and their parameters are presented in Table 1. In combination with previously published photometric data, the ASAS observations can be used to improve the precision of the binary orbital periods, by extending the baseline of the observations.

Parameters for the remaining eight objects (blue pentagons in Figure 2) are listed in Table 2, and their light curves are plotted in Figure 5. They are most probably newly discovered EB stars and we have already started follow-up spectroscopic observations in order to verify their binarity. All have OB spectral types, which is consistent with their short orbital period. Three of them had been recognized as variable stars long ago, one was misclassified as a long-period variable (052506-7127.9 $=\mathrm{Sk}$ $-7129=$ LMC V2528 ${ }^{11}$ ) by Hughes (1989), and the other two had no type specification: 051230-6727.3 = BI 98 (Butler \& Wayman 1974) and 053435-6945.7 = Sk -69 194 (Westerlund 1961).

11 "Poorly studied irregular variables of early $(\mathrm{O}-\mathrm{A})$ spectral type." GCVS. 
Table 1

Parameters of Known Binary Stars

\begin{tabular}{lccccccc}
\hline \hline \multicolumn{1}{c}{ B09 ID } & $\begin{array}{c}\text { R.A. 2000 } \\
(\mathrm{deg})\end{array}$ & $\begin{array}{c}\text { Decl. 2000 } \\
(\mathrm{deg})\end{array}$ & $\begin{array}{c}\mathrm{HJD}_{0}-2450000 \\
(\mathrm{days})\end{array}$ & $\begin{array}{c}\text { Period } \\
(\mathrm{days})\end{array}$ & $\begin{array}{c}V_{\mathrm{ASAS}} \\
(\mathrm{mag})\end{array}$ & $\begin{array}{c}\text { Amp } \\
(\mathrm{mag})\end{array}$ \\
\hline BAT99-6 (Sk-67 18) & 73.8121643 & -67.1899185 & 3060.318974 & 2.001248 & 12.04 & 0.49 & O3f* + O EB \\
BAT99-32 (Sk-71 21) & 80.5938721 & -71.5994720 & 3425.880887 & 3.815192 & 12.67 & 0.24 & WN6(h) SB \\
Sk-67 105 & 81.5257950 & -67.1824417 & 3400.010490 & 3.301529 & 12.41 & 0.43 & O4 f + O6V \\
Sk-67 117 & 81.8641281 & -67.1984711 & 3412.698954 & 4.828888 & 13.03 & 0.66 & O9 III-II + O8 V: \\
MACHO81.8763.8 & 83.6720810 & -69.5275040 & 3090.591666 & 1.404787 & 13.62 & 0.59 & O3 If* + O6:V \\
\hline
\end{tabular}

Note. ${ }^{a}$ Star designations following B09: Breysacher et al. (BAT99; 1999), Sanduleak (Sk; 1970).

Table 2

Parameters of Eight New Eclipsing Binaries

\begin{tabular}{|c|c|c|c|c|c|c|c|}
\hline B09 ID ${ }^{\mathrm{a}}$ & $\begin{array}{l}\text { R.A. } 2000 \\
\text { (deg) }\end{array}$ & $\begin{array}{l}\text { Decl. } 2000 \\
(\mathrm{deg})\end{array}$ & $\begin{array}{c}\mathrm{HJD}_{0}-2450000 \\
\text { (days) }\end{array}$ & $\begin{array}{c}\text { Period } \\
\text { (days) }\end{array}$ & $\begin{array}{l}V_{\text {ASAS }} \\
(\mathrm{mag})\end{array}$ & $\begin{array}{l}\operatorname{Amp}_{V} \\
(\mathrm{mag})\end{array}$ & Sp Type \\
\hline $\mathrm{Sk}-699$ & 72.4642105 & -69.2011948 & 3445.552851 & 1.736425 & 12.46 & 0.22 & O6.5 III \\
\hline BI 98 & 78.1266632 & -67.4539948 & 3245.926372 & 3.765188 & 14.03 & 0.62 & B1: V \\
\hline$S k-6547$ & 80.2277908 & -65.4550781 & 3585.283137 & 3.701956 & 12.41 & 0.23 & $\mathrm{O} 4 \mathrm{If}$ \\
\hline $\mathrm{Sk}-7129$ & 81.2763748 & -71.4644165 & 3462.286550 & 1.832248 & 14.04 & 0.58 & B extr \\
\hline Sk-69 194 & 83.6503296 & -69.7601395 & 3451.041843 & 12.22083 & 11.66 & 0.67 & $\mathrm{~B} 0 \mathrm{I}+\mathrm{WN}$ \\
\hline Sk-67 270 & 86.7945404 & -67.9819183 & 3474.245086 & 3.067998 & 13.74 & 0.66 & B $0.5 \mathrm{~V}$ \\
\hline
\end{tabular}

Note. ${ }^{a}$ Star designations following B09: Brunet et al. (BI; 1975), Sanduleak (Sk; 1970).

The latter, namely 053435-6945.7 (Sk -69 194), has the longest period of all EB candidates $(P=12.2$ days). According to Massey et al. (2000), it is a B0 Ia + WN star. The filling-in of the minima in its light curve (Figure 5) most probably results from the presence of a bright ( $V \approx 11 \mathrm{mag}$ ) foreground K-type star HD 269770, distant by $33^{\prime \prime}$ in the SW direction.

The remaining five EB candidates were not previously identified as variable stars. Their ASAS identifications are as follows.

1. 045617-6959.6 = Sk -70 18 (B0 n; Conti et al. 1986).

2. $044951-6912.0=\mathrm{Sk}-699$ (O6.5 III; Jaxon et al. 2001).

3. $052055-6527.3=\mathrm{Sk}-6547$ (O4 If), whose spectrum is available in the literature (Figure 7 of Massey et al. 2005). The $\mathrm{He}$ II $\lambda 4686$ and $\mathrm{H} \alpha$ lines are not well fit by the model, which may be due to wind interaction in the binary. The object was recently reclassified to $\mathrm{O} 4 \mathrm{I}(\mathrm{n}) \mathrm{f}+\mathrm{p}$ type by Walborn et al. (2010). Since O4 If stars are quite rare, measuring the parameters for this system would be valuable.

4. 052601-6703.1 = Sk-67 102 (B2 III; Conti et al. 1986).

5. $054711-6759.0=\mathrm{Sk}-67270(\mathrm{~B} 0.5 \mathrm{~V}$; Conti et al. 1986).

\subsection{Non-periodic Variables}

We identified 104 variable stars that are either semi-periodic or non-periodic. Among them are 25 variables that were already listed in the ASAS Catalog of Variable Stars (ACVS) as MISCtype variable stars, but 9 of these are not listed as variable in the Simbad database. Altogether there are 33 objects that are not marked as variable in Simbad, GCVS, or ASAS. All of them are bright and many have large light curve amplitudes, so it is noteworthy that their variability had not been discovered previously. The reason that some of them do not figure in ACVS is that the part of the catalog containing the LMC region was made when the light curves consisted of 40-50 observations only (Pojmański 2002), during which there was no significant change in the light curves.

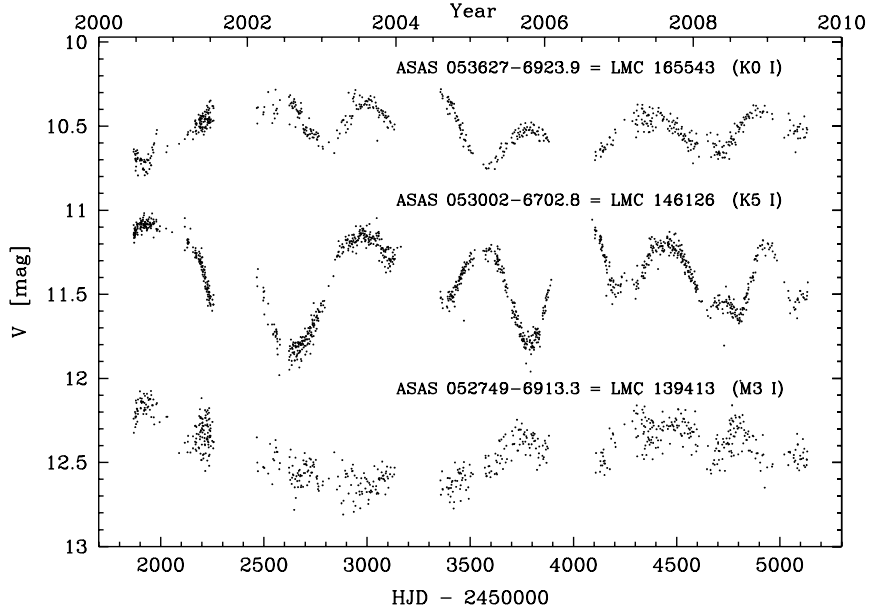

Figure 6. Light curves of 3 RSGs out of 38 objects that are newly reported as variable (ASAS 052749-6913.3 = 2MASS J05274747-6913205, ASAS 053002-6702.8 = 2MASS J05300226-6702452, ASAS 053627-6923.9= 2MASS J05362678-6923514 = CPD-69 421), spanning 10 years. The spectral type from B09 is given in brackets.

The light curves of some interesting objects that are for the first time reported as variable are plotted in Figure 6, while Figure 7 shows long-term light curves of a few known interesting variables; the last two are solely ASAS discoveries. In Figure 8, we present light curve stamps of all 104 semi- or non-periodic stars.

The parameters of all 117 variable stars, both periodic and non-periodic, are listed in Table 3, supplemented with extinction values from Pejcha \& Stanek (2009) where available (101 objects). The next to last column contains information on whether the star is a known variable (by providing its ID) or if it is a new discovery (empty field). The last column provides information about crowding problems mentioned in the fourth paragraph of Section 2.2. It contains the number of bright companions and their approximate angular distance (in arcsec) from the variable 


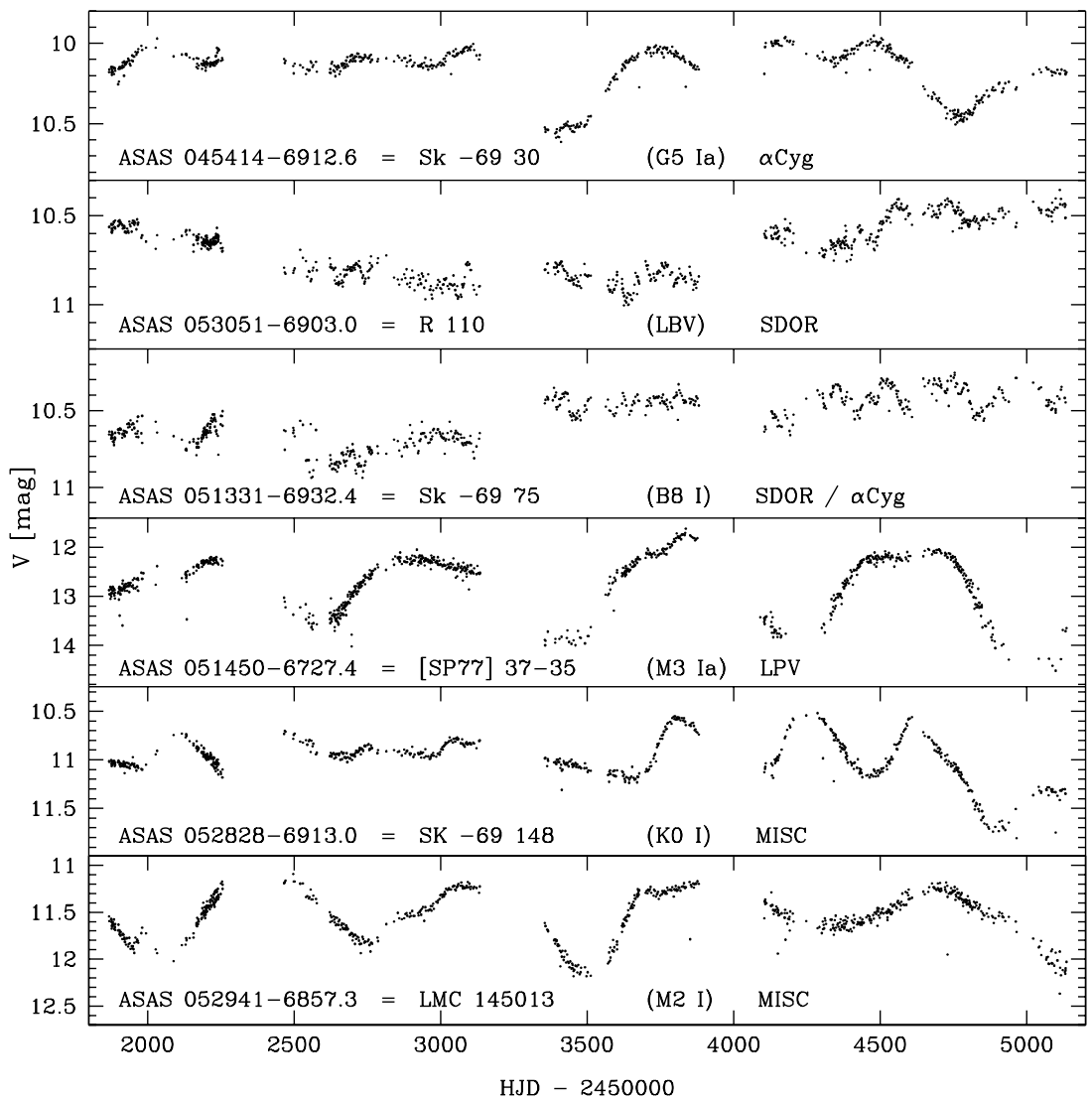

Figure 7. Ten-year long light curves of a few interesting known variable stars. The spectral type is given in brackets, followed by the variability type as found in the literature. The variability of the last two objects was discovered by ASAS (Pojmański 2002) and classified as "Miscellaneous" (MISC).

star. These objects were selected by examining the Digitized Sky Survey (DSS) images around each variable star, using the Goddard SkyView ${ }^{12}$ utility. The DSS exposures reach 2-3 mag deeper than ASAS observations, making them ideal for resolving objects otherwise indistinguishable by ASAS.

The search showed that 47 out of the 117 variables have fairly bright close companions. Usually the presence of such neighbors will be reflected in the light curves by a significant scatter in the observations, as in the case of an EB Sk -69 194 already mentioned in Section 3.2. Another two examples are RSG stars 053627-6923.9 (one bright star $\sim 10^{\prime \prime}$ away) and $052749-6913.3$ (one very bright star $\sim 20^{\prime \prime}$ away) presented in Figure 7.

Using the classification of massive stars adopted in B09 we have divided the stars in our sample into the following groups, sorted by the number of objects in the group (the abbreviation is given in brackets). For each class we list the number of variables, including periodic variables, and the total number of stars in our matched sample: early B-type stars (earlyB): 10/238, O-type stars (O): 10/156, red supergiants (RSGs): 85/97, late B-type stars (lateB): 2/51, Wolf-Rayet stars (W-R): $3 / 35$, A, F, G supergiants (AFG): $1 / 10$, supergiant $\mathrm{B}[\mathrm{e}]$ stars $(\mathrm{sgBe}): 3 / 8$, luminous blue variables (LBVs): $3 / 3$, and $\mathrm{Be} / \mathrm{X}$-ray binaries (bexrb): $0 / 1$.

As expected, RSGs constitute the majority of all variable stars $(85 / 117=73 \%)$, and they have the largest variability percentage among all classes other than the LBVs (by definition). It is also difficult to confirm that the 12 RSGs that we do not report as variable are truly not variable. Their light curves show quite

\footnotetext{
$\overline{12 \text { http://skyview.gsfc.nasa.gov/cgi-bin/query.pl }}$
}

high scatter, which could indicate lower amplitude variability than is detectable by ASAS. The percentage of variables in other classes varies from about $4 \%$ for B-type stars to about $37 \%$ for $\mathrm{sgBe}$ stars, but the number of objects in several groups is too small to perform any global statistics.

We will discuss some of these variable stars in the following section.

\section{VARIABLE STAR PROPERTIES}

\subsection{Color-Magnitude Diagrams}

In the left panel of Figure 9 we plot a CMD, showing $V$ versus $V-I$ magnitudes of all final matches between ASAS and B09 catalogs. $V$ and $I$ are the mean magnitude values based on the ASAS light curves. Gray open squares represent all 599 matches, while black dots stand for variable stars. EBs are marked with big open circles. The majority of variables have red colors, as noted in the previous section. At the same time almost all red stars turn out to be variable, which supports previous studies of RSG variability (e.g., Kiss et al. 2006, and references therein). All eclipsing variables are among the blue stars. The right panel of Figure 9 presents $V$ amplitude versus $V-I$ color for the same sample with the same designations. The amplitude range is large, from $\sim 0.1$ to $\sim 1.8 \mathrm{mag}$. Non-variable stars (gray squares) are plotted to show the scale of observational scatter in the dense regions of the LMC. For these, the amplitude $A_{\mathrm{V}}$ corresponds to the scatter in the data points, being roughly the $3 \times \sigma_{\mathrm{V}}$ value. The majority of objects with high $A_{\mathrm{V}}$ values are among the faint stars (see Figure 3).

The star with the largest amplitude value, namely ASAS $051450-6727.4=[\mathrm{SP} 77] 37-35=\mathrm{HV} 916$, is a known variable 


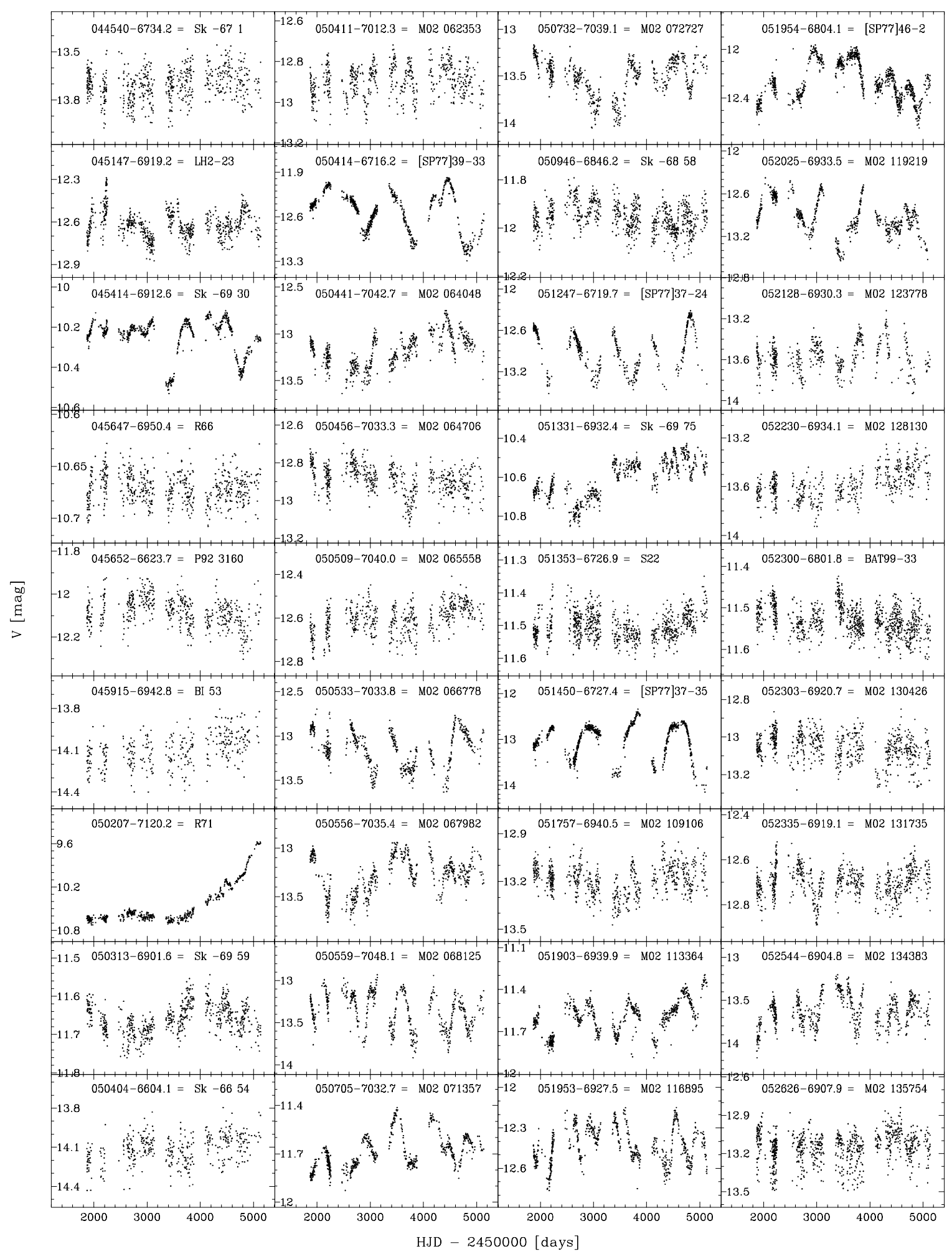

Figure 8. Light curve stamps of all 104 semi- or non-periodic variable stars identified in the course of this study.

supergiant of type M3 Ia (its light curve is plotted in Figure 7), while R71 with its dramatic rise is only fifth in amplitude and is marked with a cross in the right panel of Figure 9. The lowest amplitude variable is ASAS 045647-6950.4 = R66 which has been recognized as $S$ Dor $/ \alpha$ Cyg type variable by van Genderen $\&$ Sterken (2002) and will be discussed in Section 4.2.

Figure 10 is a CMD diagram of Spitzer/IRAC [3.6] versus [3.6]-[4.5] infrared magnitudes of the 117 variables, taken from B09 catalog. This figure is analogous to the Figure 2 of B09 where different spectral types are marked with different symbols, but here we additionally mark eclipsing variables with large open circles. Again we see that the majority of variable stars are among RSG, occupying the region of [3.6] $<9$ and [3.6]-[4.5] $<0.4 \mathrm{mag}$. All EBs are either O- or early B-type objects, except for one W-R star. 


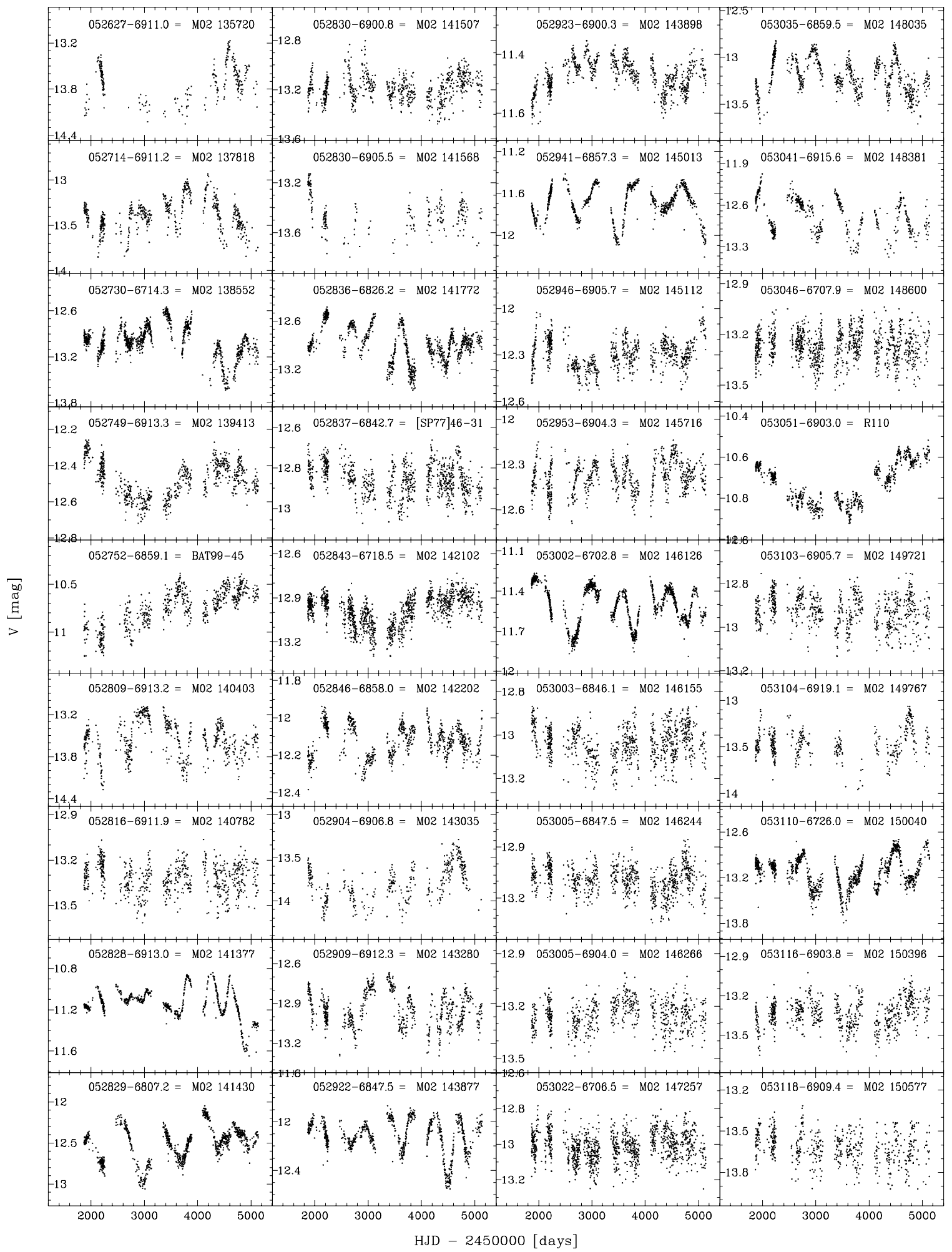

Figure 8. (Continued)

\subsection{Special Cases}

Three of the $\operatorname{sgB}[\mathrm{e}]$ stars, marked with blue diamonds in Figure 10, were previously reported as variable by van Genderen \& Sterken $(1999,2002)$ and exhibit low amplitude variability: $\mathrm{S} 22=$ HD $34664=$ ASAS 051353-6726.9 $(\sim 0.21 \mathrm{mag})$, R66 = ASAS 045647-6950.4 ( 0.08 mag $)$, and R126 =
053626-6922.9 ( $\sim 0.16 \mathrm{mag})$. We find evidence for a long-term $\mathrm{S}$ Dor-like oscillation, on the order of six years, in S22, similar to that reported by van Genderen \& Sterken (1999, p. 541), who conclude that this star "is not only a $\mathrm{B}[\mathrm{e}]$ star, but also a very weak-active LBV."

In the case of R66, van Genderen \& Sterken (2002, p. 930) observed "a long-term wave with a possible timescale 


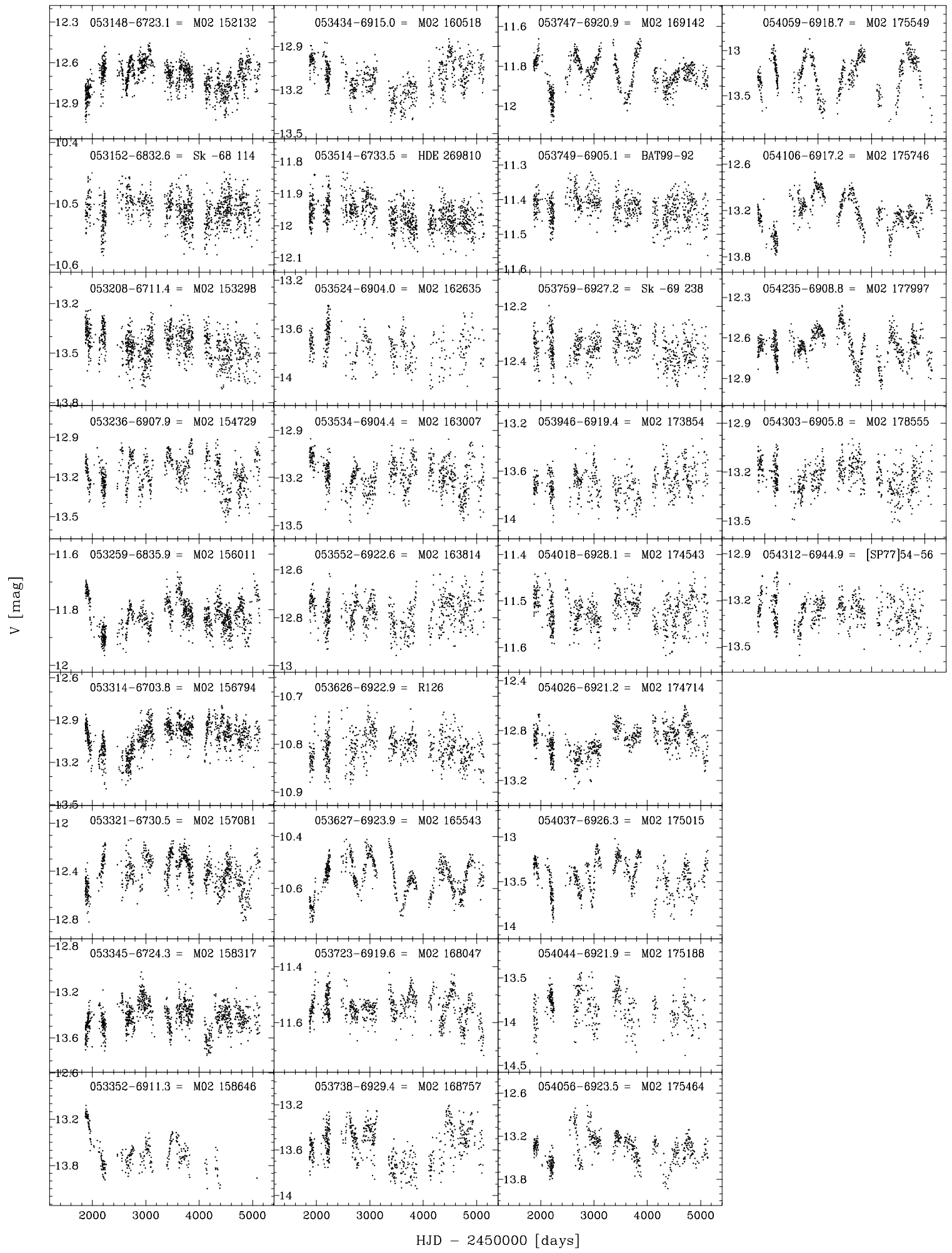

Figure 8. (Continued)

of hundreds of days and an amplitude of $\sim 0$. 06 " based on their observations from 1989 to 1991 . This is the lowest amplitude variable in our sample, with $A_{\mathrm{V}}=0.08$ being consistent with previous estimates. We observe a pulsation-type oscillation with a period of 224 days, which is roughly four times longer than the 55 day period observed by van Genderen \& Sterken (2002), attributed to $\alpha$ Cyg-type oscillations. We do not observe longer S Dor-like oscillations in this star.

\section{SUMMARY}

The aim of this paper was to investigate photometric variability of massive stars in the LMC. We performed a variability search among 599 out of 1268 objects listed in the catalog of Bonanos et al. (2009) which had their unique counterparts in the ASAS (Pojmański 2002) database, spanning 10 years of observations. We found 13 periodic and 104 semi- or non-periodic 

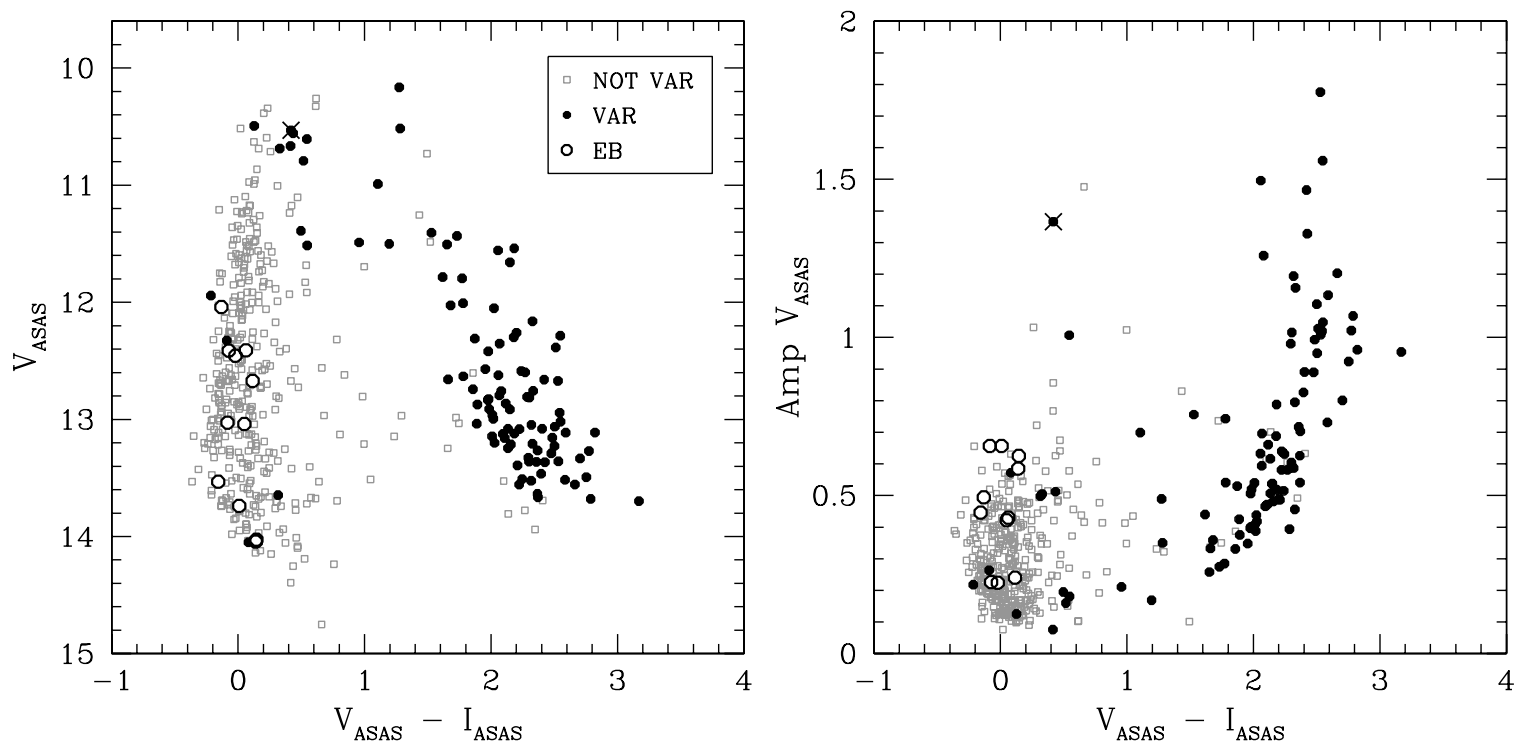

Figure 9. Left panel: CMD for the 599 matches between ASAS and B09 catalogs. $V$ and $I$ magnitudes are the mean values based on ASAS light curves. Black dots represent the 117 variable stars and gray open squares the non-variables. EBs are marked with big open circles. Right panel: $V$ amplitude of 117 variables vs. $V-I$ color with the same designations. For the non-variables, the light curve scatter is plotted. R71 is marked with a cross (both panels).

Table 3

Parameters of 117 Variable Stars Identified in this Study

\begin{tabular}{|c|c|c|c|c|c|c|c|c|c|c|}
\hline B09 ID ${ }^{a}$ & $\begin{array}{l}\text { R.A. } 2000 \\
\text { (deg) }\end{array}$ & $\begin{array}{l}\text { Decl. } 2000 \\
\text { (deg) }\end{array}$ & $\begin{array}{l}V_{\text {ASAS }} \\
(\mathrm{mag})\end{array}$ & $\begin{array}{l}\mathrm{Amp}_{V} \\
(\mathrm{mag})\end{array}$ & $\begin{array}{l}I_{\mathrm{ASAS}} \\
(\mathrm{mag})\end{array}$ & $\begin{array}{l}\mathrm{Amp}_{I} \\
(\mathrm{mag})\end{array}$ & Sp Type & $\begin{array}{c}E(V-I)^{\mathrm{b}} \\
(\mathrm{mag})\end{array}$ & Known? ${ }^{\mathrm{c}}$ & Neighbors $^{\mathrm{d}}$ \\
\hline P92 3160 & 74.2139130 & -66.3970566 & 12.03 & 0.36 & 10.35 & 0.19 & early K I & $\cdots$ & $\cdots$ & 1 bright, $\sim 25 "$ \\
\hline BI 53 & 74.8157120 & -69.7153549 & 14.04 & 0.61 & .. & .. & BO III & & $\ldots$ & 1 bright, $\sim 20 "$ \\
\hline $\mathrm{Sk}-6959$ & 75.8029175 & -69.0269470 & 11.64 & 0.20 & . & 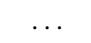 & $\mathrm{O}(\mathrm{f})+\mathrm{B} 1 \mathrm{I} ?$ & 0.000 & $\ldots$ & 1 bright, $\sim 15^{\prime}$ \\
\hline M02 062353 & 76.0413361 & -70.2049942 & 12.84 & 0.40 & 10.86 & 0.15 & M1 I & 0.120 & $\cdots$ & $\cdots$ \\
\hline M02 064706 & 76.2255402 & -70.5552521 & 12.83 & 0.40 & 10.85 & 0.19 & M0-1 I & 0.134 & $\ldots$ & $\ldots$ \\
\hline M02 065558 & 76.2917938 & -70.6675568 & 12.57 & 0.35 & 10.62 & 0.16 & M0 I & 0.000 & $\ldots$ & $\ldots$ \\
\hline $\mathrm{Sk}-6858$ & 77.4382935 & -68.7694168 & 11.92 & 0.30 & $\cdots$ & $\cdots$ & B3 Iab & 0.224 & $\ldots$ & 1 bright, $\sim 25 "$ \\
\hline Sk-69 75 & 78.3782501 & -69.5399170 & 10.56 & 0.51 & 10.12 & 0.59 & B8 I & 0.163 & V1577 & $\ldots$ \\
\hline M02 119219 & 80.0987091 & -69.5575867 & 12.76 & 1.26 & 10.68 & 0.91 & M3 I & 0.126 & V2148 & $\ldots$ \\
\hline $\mathrm{Sk}-6547$ & 80.2277908 & -65.4550781 & 12.41 & 0.23 & 12.49 & 0.27 & O4 If & 0.10 & $\ldots$ & $\ldots$ \\
\hline BAT99-32 & 80.5938721 & -71.5994720 & 12.67 & 0.24 & 12.56 & 0.37 & WN6(h) SB & 0.141 & V2296 & $\ldots$ \\
\hline
\end{tabular}

Notes.

a Star designations following B09: Breysacher et al. (BAT99; 1999), Sanduleak (Sk; 1970), Westerlund (W; 1961), Brunet et al. (BI; 1975), Lucke (LH; 1972), Henize (S; 1956), Parker et al. (P92; 2001), Massey (M02; 2002).

${ }^{\mathrm{b}}$ Mean extinction values taken from Pejcha \& Stanek (2009). Zero values (0.000) correspond to negative values for the calculated extinction $A_{I}$, which is the case for 30 out of 101 stars for which the extinction value was available.

${ }^{\mathrm{c}}$ GCVS LMC designation.

${ }^{\mathrm{d}}$ Approximate brightness and distance of close neighbors, based on DSS images. See Section 3.3 for details.

(This table is available in its entirety in machine-readable and Virtual Observatory (VO) forms in the online journal. A portion is shown here for guidance regarding its form and content.)

variables brighter than $V=14 \mathrm{mag}$, of which $\sim 32 \%$ had not been listed as variable in either Simbad, GCVS, or ASAS catalogs.

The majority of variable stars (73\%) identified are RSGs and almost all RSGs exhibit some form of variability. The variability rate among other spectral types fluctuates between $\sim 4 \%$ (for $\mathrm{O}$ and B-type stars) and $\sim 37 \%$ (for $\operatorname{sgB}[\mathrm{e}]$ stars), but the absolute numbers of stars in the groups are too small to perform reliable statistics.

All 13 periodic variables are EB stars, but to our surprise only 5 of them were known to be binary before, even though the LMC had been surveyed for bright variables about a century ago at Harvard. However, the sensitivity of the photographic plates only allowed for detection of variables with amplitudes above 0.5 mag, while all new EBs have amplitudes below 0.6 mag. The eight new EBs have $\mathrm{O}$ and B spectral types, which are consistent with their short orbital periods. We have already started follow-up spectroscopic observations in order to verify their binary nature and investigate their physical properties.

We present some of the more interesting 10 year long light curves, which for many objects are the most extensive light curves available in the literature. This is particularly important since several of the systems are relatively rare. We also provide light curve stamps for all semi- and non-periodic variables for easy reference. In addition, we present tables with the stars' parameters. All light curves and full tables are available on request. 


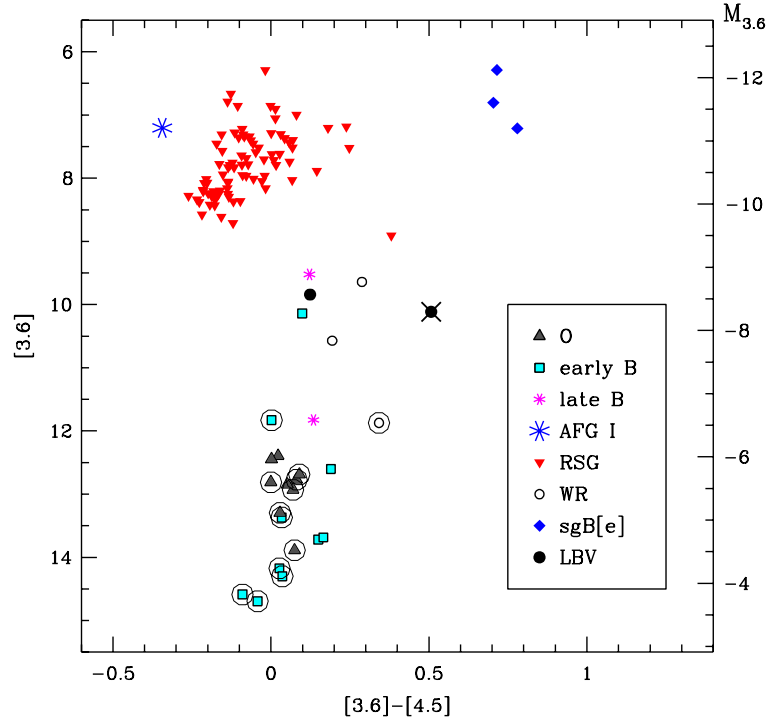

Figure 10. Infrared CMD for 113 variable stars out of 599 matches between ASAS and B09 catalogs, for which infrared colors were available. Values of [3.6] and [4.5] were taken from the B09 catalog. The designations are the same as in Figure 2 of B09. EBs are marked with big open circles and R71 is marked with a cross.

(A color version of this figure is available in the online journal.)

We are grateful to S. Kozłowski and C. Kochanek for useful discussions and a careful reading of the manuscript. We also thank the anonymous referee for suggestions that greatly improved this paper. D.M.S. and K.Z.S. are supported in part by National Science Foundation grants AST0707982 and AST-0908816. A.Z.B. acknowledges the support of the European Community under a Marie Curie International Reintegration Grant. G.P. and B.P. are supported by the Polish MNiSW grant N203 007 31/1328. J.L.P. acknowledges support from NASA through Hubble Fellowship grant HF-51261.01-A awarded by the Space Telescope Science Institute, which is operated by AURA, Inc. for NASA, under contract NAS 5-26555. This research has made use of the Digitized Sky Survey images via the Goddard SkyView utility (http://skyview.gsfc.nasa.gov/cgi-bin/query.pl), of NASA's Astrophysics Data System, as well as the SIMBAD database, operated at CDS, Strasbourg, France.

\section{REFERENCES}

Alcock, C., et al. 1995, AJ, 109, 1653

Alcock, C., et al. 1996, AJ, 111, 1146

Alcock, C., et al. 1997, AJ, 114, 326

Alcock, C., et al. 2000, ApJ, 542, 281

Bonanos, A. Z., et al. 2009, AJ, 138, 1003

Breysacher, J., Azzopardi, M., \& Testor, G. 1999, A\&AS, 137, 117

Brunet, J. P., Imbert, M., Martin, N., Mianes, P., Prévot, L., Rebeirot, E., \& Rousseau, J. 1975, A\&AS, 21, 109

Butler, C. J., \& Wayman, P. A. 1974, Dunsink Obs. Publ., 1, 193

Caldwell, J. A. R., \& Coulson, I. M. 1986, MNRAS, 218, 223

Conti, P. S., Garmany, C. D., \& Massey, P. 1986, AJ, 92, 48

Cook, K. H., et al. 1995, in ASP Conf. Ser. 83, IAU Colloq. 155, Astrophysical Applications of Stellar Pulsation, ed. R. S. Stobie \& P. A. Whitelock (San Francisco, CA: ASP), 221

Dobrzycki, A., Macri, L. M., Stanek, K. Z., \& Groot, P. J. 2003, AJ, 125, 1330 Feast, M. W., Glass, I. S., Whitelock, P. A., \& Catchpole, R. M. 1989, MNRAS, 241,375

Fiorentino, G., Contreras, R., Clementini, G., Glatt, K., Sabbi, E., Sirianni, M., Grebel, E., \& Ghallager, J. 2008, Mem. Soc. Astron. Ital., 79, 3

Freedman, W. L., et al. 2001, ApJ, 553, 47
Gamen, R., et al. 2009, IAU Circ., 9082, 1

Garg, A., et al. 2007, AJ, 133, 403

Geha, M., et al. 2003, AJ, 125, 1

Grindlay, J., Tang, S., Simcoe, R., Laycock, S., Los, E., Mink, D., Doane, A., \& Champine, G. 2009, in ASP Conf. Ser. 410, Preserving Astronomy's Photographic Legacy: Current State and the Future of North American Astronomical Plates, ed. W. Osborn \& L. Robbins (San Francisco, CA: ASP), 101

Haefner, R., Simon, K. P., \& Fiedler, A. 1994, A\&A, 288, L9

Henize, K. G. 1956, ApJS, 2, 315

Hughes, S. M. G. 1989, AJ, 97, 1634

Jaxon, E. G., Guerrero, M. A., Howk, J. C., Walborn, N. R., Chu, Y., \& Wakker, B. P. 2001, PASP, 113, 1130

Kałużny, J., Mochnacki, S., \& Ruciński, S. M. 2006, AJ, 131, 407

Kiss, L. L., Szabó, G. M., \& Bedding, T. R. 2006, MNRAS, 372, 1721

Kozłowski, S., et al. 2010, ApJ, 708, 927 (OGLE Collaboration)

Leavitt, H. S. 1908, Ann. Harvard Coll. Obs., 60, 87

Leavitt, H. S., \& Pickering, E. C. 1912, Harvard Coll. Obs. Circ., 173, 1

Leavitt, W., \& Pickering, E. C. 1905, Harvard Coll. Obs. Circ., 96, 1

Lucke, P. B. 1972, PhD thesis, Univ. of Washington

Macri, L. M., Stanek, K. Z., Bersier, D., Greenhill, L. J., \& Reid, M. J. 2006, ApJ, 652,1133

Madore, B. F., \& Freedman, W. L. 1991, PASP, 103, 933

Massey, P. 2002, ApJS, 141, 81

Massey, P., Puls, J., Pauldrach, A. W. A., Bresolin, F., Kudritzki, R. P., \& Simon, T. 2005, ApJ, 627, 477

Massey, P., Waterhouse, E., \& DeGioia-Eastwood, K. 2000, AJ, 119, 2214

Moffat, A. F. J. 1989, ApJ, 347, 373

Niemela, V. S., \& Bassino, L. P. 1994, ApJ, 437, 332

Niemela, V. S., \& Morrell, N. I. 1986, ApJ, 310, 715

Niemela, V. S., Seggewiss, W., \& Moffat, A. F. J. 2001, A\&A, 369, 544

Ostrov, P. G. 2001, MNRAS, 321, L25

Ostrov, P. G., \& Lapasset, E. 2003, MNRAS, 338, 141

Ostrov, P. G., Lapasset, E., \& Morrell, N. I. 2000, A\&A, 356, 935

Paczyński, B. 1986, ApJ, 304, 1

Parker, J. W., Zaritsky, D., Stecher, T. P., Harris, J., \& Massey, P. 2001, AJ, 121, 891

Pejcha, O., \& Stanek, K. Z. 2009, ApJ, 704, 1730

Pickering, E. C. 1904, Harvard Coll. Obs. Circ., 82, 1

Pojmański, G. 1997, Acta Astron., 47, 467

Pojmański, G. 2002, Acta Astron., 52, 397

Pojmański, G., Pilecki, B., \& Szczygieł, D. 2005, Acta Astron., 55, 275

Prevot-Burnichon, M. L., Prevot, L., Rebeirot, E., Martin, N., \& Rousseau, J. 1981, A\&A, 103, 83

Rest, A., et al. 2005, Nature, 438, 1132

Samus, N. N., et al. 2009, VizieR Online Data Catalog, 1, 2025

Sanduleak, N. 1970, Contrib. Cerro Tololo Inter-American Obs., 89

Schwarzenberg-Czerny, A. 1989, MNRAS, 241, 153

Seggewiss, W., Moffat, A. F. J., \& Lamontagne, R. 1991, A\&AS, 89, 105

Soszyński, I., Poleski, R., Udalski, A., Szymański, M. K., Kubiak, M., Pietrzyński, G., Wyrzykowski, Ł., Szewczyk, O., \& Ulaczyk, K. 2008, Acta Astron., 58, 163

Soszyński, I., Udalski, A., Szymański, M. K., Kubiak, M., Pietrzyński, G., Wyrzykowski, Ł., Szewczyk, O., Ulaczyk, K., \& Poleski, R. 2009, Acta Astron., 59, 1

Soszyński, I., et al. 2003, Acta Astron., 53, 93

Stahl, O., Wolf, B., Klare, G., Cassatella, A., Krautter, J., Persi, P., \& FerrarToniolo, M. 1983, A\&A, 127, 49

Thackeray, A. D. 1958, MNRAS, 118, 117

Tisserand, P., et al. 2007, A\&A, 469, 387

Udalski, A., Szymański, M., Kubiak, M., Pietrzyński, G., Soszyński, I., Woźniak, P., \& Żebruń, K. 1999, Acta Astron., 49, 201

Udalski, A., et al. 2008, Acta Astron., 58, 89

van Genderen, A. M., \& Sterken, C. 1999, A\&A, 349, 537

van Genderen, A. M., \& Sterken, C. 2002, A\&A, 386, 926

Walborn, N. R., et al. 2008, ApJ, 683, L33

Walborn, N. R., et al. 2010, AJ, 139, 1283

Walker, A. R. 1992, ApJ, 390, L81

Westerlund, B. 1961, Uppsala Astron. Obs. Ann., 5, 1

Westerlund, B. E., \& Smith, L. F. 1964, MNRAS, 128, 311

Wood, P. R., Bessell, M. S., \& Fox, M. W. 1983, ApJ, 272, 99

Wyrzykowski, Ł., Udalski, A., Kubiak, M., Szymański, M. K., Żebruń, K., Soszyński, I., Woźniak, P. R., Pietrzyński, G., \& Szewczyk, O. 2004, Acta Astron., 54, 1

Wyrzykowski, Ł., et al. 2009, MNRAS, 397, 1228

Żebruń, K., et al. 2001, Acta Astron., 51, 317 